\title{
Tacto Pedagógico y enseñanza de cuentos tradicionales en estudiantes de Primaria distrito Huayllabamba Sihuas, Ancash 2019.
}

\author{
Pedagogical touch and teaching of traditional tales in súdenos of Primary Huayllabamba Sihuas \\ district, Anacash 2019. \\ Fortunato Diestra Salinas ${ }^{1}$ Virginia Asunción Cerafin Urbano ${ }^{1}$ Mara Sandra Rivera Galindo² César Julián Contreras Limache ${ }^{3}$ \\ Henry Nickeil Díaz Deggola ${ }^{2}$
}

\begin{abstract}
RESUMEN
Objetivo: Identificar la relación que existe entre el tacto pedagógico y la enseñanza de cuentos tradicionales en estudiantes de Primaria perteneciente al distrito de Huayllabamba Sihuas, región Áncash 2019. Métodos: Es un estudio de tipo básico, de nivel descriptivo correlacional, trabajado con una muestra de 60 docentes del nivel de educación primaria en el ámbito del distrito, se utilizó como instrumentos 2 cuestionarios de 20 preguntas cada uno. Para la prueba de normalidad se aplicó Kolmogorov-Smimov, que nos indicó que la población tiene una distribución no normal, por lo que se utiliza pruebas no paramétricas como es Rho de Spearman. Resultados: El 33,3\% de los docentes manifestaron que el tacto pedagógico es bajo, mientras un 58,3\% lo catalogaron como regular y solamente el $8,3 \%$ lo consideraron en el nivel bueno, lo que significa, que los docentes deben tomar algunas estrategias para poder mejorar este impase hasta llegar al nivel alto. En la variable enseñanza de cuentos tradicionales, el $30,3 \%$ de encuestados manifiesta que la enseñanza de cuentos tradicionales es bajo, un $61,3 \%$ sostiene que es regular, mientras el $8,4 \%$ asevera que el nivel es alto. Conclusión: se llegó a la conclusión que sí existe relación positiva de alta confiabilidad, siendo la correlación de 0,892 entre el tacto pedagógico y la enseñanza de los cuentos tradicionales.
\end{abstract}

Palabras Clave: Tacto pedagógico y enseñanza de cuentos tradicionales.

\section{ABSTRACT}

Objective: Identify the relationship between pedagogical tact and the teaching of traditional stories in Primary students belonging to the district of Huayllabamba Sihuas, Ancash region 2019. Methods: It is a basic type study, descriptive correlational level, worked with a sample of 60 teachers of the primary education level in the district, it was used as instruments 2 questionnaires of 20 questions each. For the normality test, Kolmogorov-Smimov was applied, which indicated that the population has a non-normal distribution, so non-parametric tests such as Spearman's Rho are used. Results: $33,3 \%$ of the teachers stated that the pedagogical touch is low, while $58,3 \%$ classified it as fair and only $8,3 \%$ considered it at the good level, which means that teachers must take some strategies to improve this impasse until you reach the high level. In the variable teaching traditional stories, $30,3 \%$ of respondents state that the teaching of traditional stories is low, $61,3 \%$ maintain that it is regular, while $8,4 \%$ assert that the level is high. Conclusion: It was concluded that there is a positive relationship of high reliability, with a correlation of 0,892 between pedagogical tact and the teaching of traditional stories.

Key Words: Pedagogical touch and teaching of traditional hundreds.

\section{INTRODUCCIÓN}

La ciencia de la educación en nuestros tiempos como toda ciencia sufre modificaciones debido al adelanto de la tecnología educativa. Ello da motivo para que el docente se encuentre preocupado por hacer uso de ciertas estrategias didácticas a fin de poder impartir una enseñanza adecuada, clara, pertinente y precisa a satisfacción de los estudiantes. Una de estas estrategias es contar con una cualidad llamada paciencia y empatía, a fin de brindar al estudiante un buen trato y entender la realidad que lo rodea, ello para poder darle la orientación respectiva escogiendo un camino adecuado y con asertividad a fin de que pueda orientarse y seguir el proceso de aprendizaje que le corresponde lograr durante el desarrollo de las clases del docente.

Esta forma de proceder en la convivencia diaria con el estudiante, para formar el carácter, basado en la práctica de valores, como la tolerancia es llamada también tacto pedagógico, mediante ello el docente maneja ésta sabiduría considerando en que momento debe actuar y en qué momento no, si es preciso y necesario actuar como una orientación consciente en cuanto a la forma de ser y actuar con los niños o adolescentes que están en formación.

Van Manen (1998) manifestó que: Todos sabemos algo sobre el tacto y sobre la necesidad de usarlo en nuestro trato con los niños. Las teorías de la educación sorprendentemente, guardan silencio respecto a la importancia de la calidad del tacto y sus manifestaciones. (p. 149), es necesario entender cuando se dice que, en ciertos modos es preciso actuar y no actuar, lo que significa que el docente en su actuación tiene que ser eficaz, actuar cuando el estudiante lo requiera es decir

Recibido 01/09/2020 Aprobado 15/09/2020

${ }^{1}$ Universidad Nacional José Faustino Sánchez Carrión de Huacho - Perú

${ }^{2}$ Universidad Privada del Norte Lima-Perú.

${ }^{3}$ Escuela de Sub - Oficiales de la Policía Nacional del Perú Alipio Ponce Vásquez Lima - Perú.

Big Bang Faustiniano revistas.unjfsc.edu.pe:

Julio - Setiembre 2020

pag.19 
actuar con precisión para tratar de ayudar en la solución de algún problema, cuando el estudiante está molesto sería conveniente no actuar y por el contrario esperar estratégicamente que pase el mal momento para poder conversar, y en ese diálogo tratar de orientarlo para que mejore en su comportamiento, convivencia con sus compañeros y demás personas. Como docente es necesario tener paciencia sabiendo que es una virtud fundamental de todo educador.

Por su parte García (2013) define el tacto educativo como sentimiento de amor el desear y procurar el bien para alguien" (p.88), es por ello que, el docente tiene que cumplir su labor docente aplicando como estrategia el entendimiento, el buen trato, buscando que todo ello esté dirigido a formar al estudiante principalmente su carácter, modulando su comportamiento, teniendo en cuenta que cuando sea adulto será un padre de familia consciente responsable de sus actos y como ciudadano practicar los buenos modales.

Van Manen (1998), consideró que "el carácter es la identidad distintiva que marca al individuo" (p.195), es entendible entonces que el carácter que se forma en el estudiante debe ser desde el inicio, ya que esta formación va a servir de sustento de su comportamiento del estudiante en todo los niveles educativos, incluyendo la vida Universitaria, y posteriormente mantenga este carácter cuando sea profesional, padre de familia y ciudadano. Sin embargo Domínguez (2011) complementa lo expresado anteriormente sosteniendo que : "la identidad constituye su núcleo más profundo y comprende el conjunto de rasgos y cualidades que la persona reconoce como propios, entendidas como las potencias físicas y psíquicas" (p.62), como es correcto entender, la identidad de la persona es importante, mediante ella el estudiante descubre de donde viene, que realidad social le cobija, y dentro de qué tipo de sociedad vive, entender además que dentro de ello tiene que desarrollar y crecer sus costumbres y el folclor del pueblo donde vive, en ciertos casos practicando una danza, una canción, poesía, conociendo o degustando su gastronomía y utilizando, valorando y conociendo su vestimenta toso ello implica un gran compromiso e identificación.

Cuento: De acuerdo a su definición existen varios estudiosos que dan su punto de vista al respecto entre ellas podemos citar a:

Ruiz (2000) quien definió al cuento infantil como un conjunto de expresiones con escenarios descritos muy brevemente, donde los personajes tienen ciertas características que cumplir realizando acciones claras para el entendimiento del niño o niña, llegando a un final adecuado de los hechos, es decir los cuentos se irán adecuando de acuerdo a la edad de quien lee, teniendo en cuenta que cada edad necesita una motivación diferente (p56).

Por su parte Jiménez, (2016) manifestó que: los cuentos tienen un estímulo positivo de aprendizaje, y colaboran con el desarrollo de los estudiantes, enriqueciendo la lectura y la escritura como medio y estrategia dinámica, creativa e integrada en su actividad diaria de clases. Por eso recomendó el uso del cuento como valor educativo el mismo que permitirá la motivación y el aprendizaje en los estudiantes de tercer grado de primaria logrando así no solo competencias en el área de Conocimiento del Medio sino también afianzar la creatividad y la producción futura de escritos (p.314).Cabe agregar al respecto que el distrito de Huayllamaba es un pueblo conformado por varios pueblos del campo y, en ellas existen muchos cuentos tradicionales, que los padres de familia o personas mayores como los abuelos de muchos niños acostumbran contar en forma oral, pasando esta de generación en generación; contribuyendo con ello los docentes quienes hacen uso de ello, como material didáctico, logrando con el trascurrir del tiempo que se vayan formando la identidad de los estudiantes al conocer que en su comunidad existen cuentos famosos que tienen trascendencia evidenciándose a través de títulos como Curupa human, ( cabeza de Gusano) Chuquijambo, el achcay y otros que hoy en día conforman la galería de cuentos famosos creados en el distrito por sus antepasados actualmente guardados y relatados como reliquias de sus antepasados.

\section{MATERIAL Y MÉTODOS}

Corresponde al tipo de estudio aplicado, de acuerdo a Caballero (2019) es de nivel descriptivo correlacional, por que busca encontrar el grado de correlación existente en las variables tacto pedagógico y el aprendizaje del cuento tradicional en el distrito de Huayllabamba Sihuas Ancash 2019. Se trabajó con 60 docentes de las diferentes Instituciones Educativas del nivel de educación primaria del distrito en mención donde se centró nuestra investigación. El estudio realizado es de forma transaccional porque los datos se recogieron, e interpretaron en un mismo momento, con el apoyo de la estadística descriptiva e inferencial, para interpretar los resultados que se obtienen como producto de la investigación. Como instrumento se utilizaron dos cuestionarios conformado por 20 preguntas cada uno, con los cuales se recogió la información. previamente los instrumentos fueron validados por juicio de expertos, posteriormente se obtuvo la fiabilidad mediante el alfa de Crombach. 


\section{RESULTADOS}

\section{Tabla 1.}

Frecuencia y porcentaje de las variables tacto pedagógico y Cuentos tradicionales y sus dimensiones

\begin{tabular}{|c|c|c|c|}
\hline Variables & Dimensiones & $\begin{array}{l}\text { Valor/ } \\
\text { Rango }\end{array}$ & Porcentaje \% \\
\hline \multirow[t]{12}{*}{ Tacto Pedagógico } & \multirow[t]{3}{*}{ Tacto pedagógico } & Bajo & $33,3 \%$ \\
\hline & & Regular & $58,4 \%$ \\
\hline & & Alto & $8,3 \%$ \\
\hline & \multirow[t]{3}{*}{ Educación paternal } & Bajo & $31,7 \%$ \\
\hline & & Regular & $56,7 \%$ \\
\hline & & Alto & $11,7 \%$ \\
\hline & \multirow[t]{3}{*}{ Educación escolar } & Bajo & $28,3 \%$ \\
\hline & & Regular & $35 \%$ \\
\hline & & Alto & $36,7 \%$ \\
\hline & \multirow[t]{3}{*}{ Experiencia docente } & Bajo & $16,7 \%$ \\
\hline & & Regular & $51,7 \%$ \\
\hline & & Alto & $31,7 \%$ \\
\hline \multirow{7}{*}{$\begin{array}{l}\text { Cuentos } \\
\text { tradicionales }\end{array}$} & \multirow[t]{3}{*}{ Cuentos Tradicionales } & Bajo & $30,3 \%$ \\
\hline & & Regular & $61,3 \%$ \\
\hline & & Alto & $8,4 \%$ \\
\hline & \multirow[t]{2}{*}{ Emociones } & $\begin{array}{l}\text { Bajo } \\
\text { Regular }\end{array}$ & $\begin{array}{c}56,7 \% \\
40 \%\end{array}$ \\
\hline & & Alto & $3,3 \%$ \\
\hline & identidad & $\begin{array}{l}\text { Bajo } \\
\text { Regular } \\
\text { Alto }\end{array}$ & $\begin{array}{l}28,3 \% \\
33,4 \% \\
38,3 \% \\
\end{array}$ \\
\hline & Imaginación & $\begin{array}{l}\text { Bajo } \\
\text { Regular } \\
\text { Alto }\end{array}$ & $\begin{array}{l}16,7 \% \\
51,7 \% \\
31,6 \%\end{array}$ \\
\hline
\end{tabular}

En los resultados de la tabla 1 se pueden observar la opinión de cada uno de los encuestados quienes manifiestan su sentir tanto en cada variable así como en las dimensiones. La prueba de normalidad de Kolmogorov - Smirnov indica que el resultado de ambas variables no sigue una distribución normal, en ambos casos siendo $(p<0,05)$, permitiendo de esta manera hacer uso de prueba no paramétrica, utilizando por consiguiente la correlación Spearman. El coeficiente de Spearman indica que existe relación entre el tacto pedagógico y la enseñanza de los cuentos tradicionales, siendo el hallazgo de correlación de alta confiabilidad de
0,892 , donde $(p<0,000)$, mediante el cual se confirma que el tacto pedagógico se relaciona en forma directa con la enseñanza de los cuentos tradicionales. En ese mismo sentido en cada uno de los hipótesis específicas que conforman el tacto pedagógico con las dimensiones de cuentos tradicionales. Es así que, el coeficiente de correlación del tacto pedagógico con la dimensión emociones fue de 0 . , 764, lo que significa una correlación directa de moderada confiabilidad ( $p<$ 0,000 ) afirmando que, el tacto pedagógico se relaciona directamente con la dimensión emociones, que se demuestra en la tabla 2. 


\section{Tabla 2}

Coeficiente de correlación de Spearman entre el tacto pedagógico y la enseñanza de cuentos tradicionales, sus dimensiones emociones, identidade imaginación.

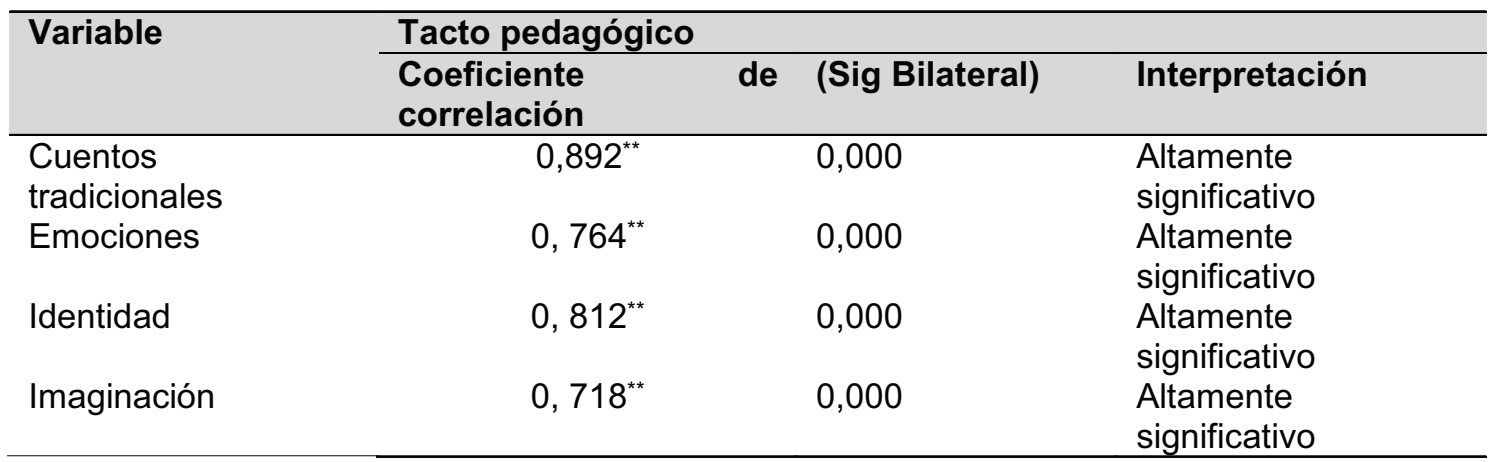

\section{DISCUSIÓN}

Las investigaciones que hemos tenido en cuenta, y que nos han servido como antecedentes referente al tema que hemos tratado, tienen ciertas similitudes con el nuestro de tal manera que, coincidimos con el de García (2013), quien al realizar el estudio del tacto pedagógico manifestó que, es el sentimiento de amor de aplicar como el desear y procurar el bien para alguien", lo que coincidimos al considerar que el docente es el formador de la sociedad, por consiguiente debe formar dentro de la práctica de valores teniendo en cuenta que se busca formar una sociedad justa, participativa y crítico que, aprenda a convivir en paz con los demás, y el docente debe enseñar con su práctica como el ejemplo, en esa misma línea Jiménez, (2016) concluye que los cuentos tienen estímulo positivo de aprendizaje, desarrollando las habilidades de los estudiantes propiciando la creatividad y la producción de futuros escritos, lo que coincidimos plenamente, porque en nuestro trabajo encontramos que el $38,3 \%$ de los encuestados manifiestan que forman la identidad del estudiante, acción de suma importancia para nosotros, porque la persona debe iniciar valorando primero lo que tiene dentro de su pueblo o comunidad.

Nuestro estudio está enfocado a entender cómo el docente hace uso de la importancia a los cuentos tradicionales que existen dentro del distrito de Huayllabamba, comprendiendo como docente que, los recursos existentes dentro de las comunidades o pueblos son elementos importantes para utilizarlos como material didáctico y que muy bien se adecuan a la realidad del alumno, despertando el interés por conocer de lo que tienen y el significado que tiene, como tal convirtiéndose en material motivador para el aprendizaje efectivo.

Los resultados de nuestra investigación, nos dan una clara evidencia al realizar la entrevista referente a la variable tacto pedagógico, encontramos que, de los 60 encuestados, el 33,3\% manifiesta que el tacto pedagógico es bajo, el $58,3 \%$ de los encuestados lo ubican en el nivel regular y solamente un $8,3 \%$ lo coloca en el nivel bueno, lo que significa, que los docentes deben tomar algunas estrategias para poder mejorar este impase hasta llegar al nivel alto. De la misma manera en la variable enseñanza de cuentos tradicionales, se observa que, el $30,3 \%$ de los encuestados manifiesta que la enseñanza de cuentos tradicionales es bajo, el 61 de los encuestados manifiestan que es regular, el $8,4 \%$ de los encuestados manifiestan que es alto, lo que significa que los docentes en su gran mayoría enseñan en forma regular, lo que significa utilizar estrategias para mejorar este impase. Al realizar la prueba de hipótesis se verifica que, la correlación de la variable tacto pedagógico y enseñanza de cuentos tradicionales es de alta confiabilidad de 0,892 , verificándose que las hipótesis especificas tienen correlaciones de 0,$764 ; 0,812$ y 0,718 .

\section{CONCLUSIONES}

Los resultados de nuestro estudio nos permiten concluir que:

Los cuentos tradicionales en el distrito de Huayllabamba, se hacen uso de forma regular, no dando importancia a la significancia como material didáctico que tiene para la población estudiantil del lugar, debido a que es formador de la identidad del estudiante, al valorar el significado que tienen estos cuentos que son herencia de sus antepasados sumado a que estas creencias a través del cuento se trasmiten de generación en generación en forma oral. Que al identificar de donde son estos cuentos tradicionales están identificándose con su pueblo, al valorar ello reconoce lo que tiene en su comunidad, acto que le permite identificarse o como tal, saber que es parte de su identidad, evidencia que lo demuestra la encuesta en la que se manifiesta que el $38.3 \%$ de encuestados lo ubican en el nivel alto.

El personal docente, no tiene idea clara que el uso de los recursos existentes en las comunidades es de importancia para el aprendizaje de los estudiantes de acuerdo a su realidad, es necesario tomar en cuenta esta opción a fin de mejorar el trabajo pedagógico, considerando que es más efectivo lograr aprendizajes óptimos que siempre permanecen en la memoria, además teniendo en cuenta que los cuentos en su contenido traen mensajes claros que sirven para 
interpretar, y aprender a razonar, desarrollando de esa manera la imaginación del estudiante.

\section{REFERENCIAS BIBLIOGRAFICAS}

Caballero. A. (2009): Innovaciones en las guías metodológicas para los planes y tesis de maestría y doctorado. (2da e.d). Lima: Instituto metodológico ALEN CAROI.

Domínguez, Xosé (2011). Ética del docente. Madrid: Fundación Emmanuel Mounier.

García, M. (2013). En el aula y fuera de ella: Educar con la vida. Edetania, No 43.

Jiménez, B (2016) Aprendizaje y rendimiento académico a través de cuentos pedagógicos (tesis de posgrado) Universidad Complutense de Madrid.
Revista Dilemas Contemporáneos: Educación, P o I ít i c a y a l or e s. http://www.dilemascontemporaneoseducacionpoliticayv alores.com/ ISSN: 2007 - 7890. Año: IV. Número: 2. Artículo no.22 Período: Octubre, 2016 - Enero, 2017.

Ruiz, A. (2000). Literatura Infantil: introducción a su teoría y práctica. Sevilla: Guadalmena.

Van Manen, M. (1998) El tacto en la enseñanza. El significado de la sensibilidad pedagógica. Barcelona: Paidós Educador. 